\title{
New considerations for the cognitive locus of impairment in the irrelevant-sound effect
}

\author{
Kirk A. Stokes • Karen M. Arnell
}

Published online: 28 February 2012

(C) Psychonomic Society, Inc. 2012

\begin{abstract}
The finding that serial recall performance for visually presented items is impaired by concurrently presented taskirrelevant speech or sounds is referred to as the irrelevantspeech/-sound effect (ISE). Substantial evidence has indicated that the impairment of serial rehearsal can result in an ISE, and this may be explained by several models. The present series of experiments has demonstrated an ISE in surprise nonserial recognition tasks in which participants were unaware of the need to maintain a large number of visual items for a later memory test, suggesting that neither the rehearsal nor maintenance of order information is necessary for observing the ISE. This effect was observed for both steady-state and changingstate irrelevant sounds, suggesting that the present results do not derive from a confusion of order information, but instead provide evidence that identity representations can also be impaired by irrelevant sound.
\end{abstract}

Keywords Irrelevant-sound effect · Recall · Recognition · Memory

\section{Introduction}

We might think that we can choose to ignore material that we know to be irrelevant. However, auditory material that is known to be irrelevant has consistently been shown to reduce memory performance for visually presented items, even if the auditory material has no meaningful semantic content. This effect is known as the irrelevant-speech effect,

K. A. Stokes $\cdot$ K. M. Arnell $(\bowtie)$

Department of Psychology, Brock University,

500 Glenridge Avenue,

St. Catharines, Ontario L2S 3A1, Canada

e-mail: karnell@brocku.ca or more recently, the irrelevant-sound effect (ISE; Colle \& Welsh, 1976; Jones \& Macken, 1993).

The prototypical ISE study requires participants to attend to short (e.g., 8-12 item) lists of visually presented items (typically digits or letters) presented under conditions of silence or with concurrent irrelevant speech or sounds, and then to recall the visual items immediately, in the correct order, under conditions of silence. Colle and Welsh (1976) were the first to demonstrate that concurrent presentation of irrelevant (to-be-ignored) speech impairs immediate serial recall of visually presented verbal material. In their study, participants were presented with serial lists of eight random visual consonants. Irrelevant speech was spoken in German - a language unfamiliar to all participants in the study. The participants showed lower serial recall scores for consonants presented at the same time as irrelevant speech, as compared to serial recall scores for consonants presented during silence. Since that first demonstration, the ISE has been observed with various to-be-remembered visual items, such as consonants, digits, or words (e.g., Beaman \& Jones, 1997; LeCompte, 1994), and with a variety of irrelevant changingstate sounds, such as auditory tones that vary unpredictably in pitch or rhythm (Jones \& Macken, 1993).

Several theoretical models have been applied to explain the ISE. Some of these models - for example, the object-oriented episodic record model (O-OER; Jones, 1993) and the primacy model of Page and Norris (1998) - suggest that disruptions of serial order information underlie the ISE. Other models, which focus more on phonological interference (e.g., the feature model of Neath, 1999) or on the attentional disruption of identity codes (e.g., the embedded-process model of Cowan, 1995, 1999, or the model of Broadbent, 1984), propose no role for serial rehearsal and the impairment of order information. A third category of models, such as the more recent perceptual-gestural account of Jones and colleagues (e.g., Jones, Hughes, \& Macken, 2006; Jones, Macken, \& Nicholls, 
2004) and the duplex-mechanism model (e.g., Hughes, Vachon, \& Jones, 2005), conceptualise serial order impairment as one of two possible processes with which irrelevant auditory information can interfere (i.e., disruption can occur during the process of organising incoming perceptual stimuli or during the planning of the output).

\section{An ISE without serial recall?}

The vast majority of experiments examining the ISE have utilised serial recall tasks with short sets of known items (e.g., Colle \& Welsh, 1976; Jones \& Macken, 1993). These findings provide evidence that irrelevant sound can impair the maintenance of serial order information. However, it is less clear whether irrelevant sound can impair the identity information of the to-be-remembered material (i.e., does irrelevant sound just impair knowing that the 2 came after the 4 , or can it also impair knowing that there was a 2 or a 4?). A handful of studies have addressed this issue by examining the ISE in free recall or recognition tasks. The first test of whether or not serial rehearsal is required for observing the ISE came from Salamé and Baddeley (1990), who examined the effect of irrelevant speech in an immediate free recall paradigm. Participants did not show an ISE when they were permitted to report 16-item visual word lists in any order they wished.

LeCompte (1994) demonstrated that the ISE is not necessarily limited to tasks that require the maintenance of order information by finding the ISE in tasks of free recall and recognition. In a test of free recall, participants viewed 12 letters on a computer screen under conditions of irrelevant speech, white noise, or silence. One second later, participants recalled the letters in any order they wished. Irrelevant speech significantly impaired recall performance compared to a silent control condition. In a separate recognition task, participants viewed words presented one at a time in the presence of irrelevant speech or of a continuous steady-state white noise stream. During the test phase, participants viewed words one at a time in silence and indicated whether each word was old (was shown in the study phase) or new (was not shown in the study phase). Results showed significant recognition memory impairment for items presented during speech versus white noise. A similar experiment showed an ISE with a two-alternative forced choice recognition memory test in which participants reported which of two words had been presented in the study phase. In the final experiment in the series, LeCompte (1994) had participants study pairs of words. At test, one word from the pair was presented, and participants were asked to type the paired associate from the study list. This paired-associate task also showed a small but significant ISE.

After finding the ISE in several nonserial tasks (free recall, cued recall, and recognition) LeCompte (1994) argued that maintenance of order information was not necessary for finding the effect. Instead, he discussed the notion that auditory stimuli have priority in short-term memory, such that the most recent auditory material, whether relevant or irrelevant, will tend to impose itself over visually presented material or previously presented auditory material.

Although LeCompte's (1994) results appear compelling in terms of ruling out serial order confusion as a basis for the ISE, it is important to note that, in all conditions, participants were fully aware that their memory for the visual items would be tested. Given the relatively short list length, and the fact that memory was tested after each list, participants may have employed a serial rehearsal strategy even though it was not explicitly required for completing the task (Beaman \& Jones, 1997). Thus, it is not clear whether the ISE observed in LeCompte's (1994) experiments was or was not a result of interference with the serial order information during rehearsal.

To examine whether or not participants rehearsed the lists while performing LeCompte's (1994) tasks, Beaman and Jones (1997) performed a series of experiments designed to replicate the approach used by LeCompte, with one key distinction: Participants were given the additional task of articulating a short series of letters throughout each set. This concurrent articulation is thought to suppress the process of serial rehearsal, such that irrelevant speech/sound cannot additionally interfere with the rehearsal of to-be-remembered items (Beaman \& Jones, 1997). When Beaman and Jones had participants engage in this additional articulation task during LeCompte's (1994) free recall and recognition tasks, the ISE disappeared. Beaman and Jones argued that the addition of concurrent articulation would not have eliminated the ISE unless participants had been rehearsing the words during the recognition and free recall tasks.

Although the results of Beaman and Jones (1997) cast doubt on LeCompte's (1994) experiments as examples of an ISE without serial rehearsal, the results do not rule out the possibility that the ISE could be observed without serial rehearsal or the need to maintain order information. Despite the fact that the ISE is often explained by the disruption of order information maintained during serial rehearsal, currently there is no strong evidence for or against the idea that disruption of rehearsal and order information is required for showing an ISE or that irrelevant sound may impair identity codes in addition to order information. Deciding whether or not impairment of serial rehearsal is required for the ISE has been difficult because whenever participants know that their memory for presented material will be tested, they may engage in serial rehearsal, even if the task itself does not require such rehearsal (Beaman \& Jones, 1997).

In this series of experiments, we tested whether the ISE could be observed in a surprise nonserial recognition paradigm in which memory for order information was not required and rehearsal was very unlikely to take place. Experiment 1 was a conceptual replication of LeCompte (1994) in which participants performed a nonsurprise, nonserial recognition 
memory task for words that were presented under conditions of silence or of changing-state tones. Experiment 2 was based on a task identical to the nonserial recognition task of Experiment 1, with the exception that participants were not told that they would need to remember the items (i.e., a surprise recognition memory task). Experiment 3 was a replication of Experiment 2 using foreign speech as the irrelevant sound. Experiment 4 contrasted recognition memory performance between changing- and steady-state irrelevant sounds versus silence.

Just giving participants a list of words at study, even without instructions to remember them, is likely to invoke suspicion as to the possibility of an impending memory test. To remove this suspicion, we used the novel approach of presenting the to-be-remembered words at study in a single, 200 -item list as part of a lexical decision task. The use of such a large word list and the lexical decision cover task should have all but eliminated the likelihood that participants would rehearse the words. The inclusion of the lexical decision task was pivotal to the design of Experiments 2-4, in which participants were not informed about the subsequent recognition memory test until after completing all lexical decision trials. However, the lexical decision task was also used in Experiment 1, in which participants were informed about the impending recognition memory test, given that Experiments 1 and 2 would then be identical except for the element of surprise.

\section{Experiment 1}

Experiment 1 tested whether memory for item order is necessary for observing the ISE by examining the ISE under conditions in which participants were free to report items in any order on a recognition test. If processes responsible for maintaining order representations do underlie the ISE, then to-beremembered visual items presented with concurrent, irrelevant changing-state auditory items should be equally likely to be reported as to-be-remembered visual items presented under a silent control condition. However, if order representations do not underlie the ISE, an ISE might still be observed in the nonserial recognition memory paradigm.

To ensure that the irrelevant-sound conditions were also capable of producing an ISE in a more typical task, the same participants also completed a serial recall task in which nine-item to-be-remembered consonant lists were to be recalled in the same order in which they were presented.

\section{Method}

Participants Thirty-two undergraduate students (20 female, 12 male) at Brock University participated in this experiment. Their ages ranged from 18 to 22 years, with a mean of 19.06 years. In each of the experiments reported herein, all participants reported normal or corrected-to-normal visual acuity, and none reported auditory impairments. Participants were compensated with either a small honourarium or research hours for a course.

Stimuli All experimental stimuli were presented, and responses collected, using a Sony VAIO desktop computer running E-Prime (Version 1.1; Schneider, Eschman, \& Zuccolotto, 2002). Visual stimuli were located centrally and presented in 18-point black Courier font on a white background using a 17-in. CRT monitor with a refresh rate of $75 \mathrm{~Hz}$. Word stimuli used in the recognition memory task were approximately $1 \mathrm{~cm}$ high and $3-5 \mathrm{~cm}$ wide. At an unfixed viewing distance of approximately $50 \mathrm{~cm}$, the visual angles were $1.2^{\circ}$ in height and $3.4^{\circ}-6.0^{\circ}$ in width. The letter stimuli used in the serial recall task were approximately $1 \mathrm{~cm}$ high and wide, for a visual angle of $1.2^{\circ}$ in height and width.

The MRC Psycholinguistic Database (www.psy.uwa.edu. au/mrc_database/uwa_mrc.htm; Kučera \& Francis, 1967) was used to gather a pool of 720 monosyllabic words for use in the recognition memory portion of the experiment. All of the words were four to six letters in length, were emotionally neutral, and were of moderate to high familiarity and concreteness. A second pool of 256 phonotactically correct pseudowords (e.g., GRONK) four to six letters in length were also generated. Pseudowords were designed such that all phonetic and morphemic rules of English were obeyed. None of the pseudowords were homophones of real words (i.e., none had the phonology of a real word-e.g., "phox"). A total of 100 words and 100 pseudowords were selected randomly from these pools and presented to each participant during the lexical decision phase of the experiment ( 25 additional words were selected randomly for presentation as foils during the recognition test phase). A given word was selected and displayed only once throughout each session, and selection was randomised for each participant.

The visual stimuli for the serial recall task were nine randomly selected consonants (K, V, S, M, L, Q, T, R, and F). Each of these consonants was presented once on each trial, in random order. All consonants were presented centrally in capital form in black 18-point Courier font.

For both the recognition memory task and the serial recall task, the irrelevant auditory stimuli were 44 nonsinusoidal square waveforms, each $400 \mathrm{~ms}$ in duration. Tones were presented at a rate of two per second (interstimulus interval = $100 \mathrm{~ms}$ ). Tones ranged in frequency (pitch) from 83 to $740 \mathrm{~Hz}$, equating to a span of three octaves in Western music. Each tone was separated by one musical semitone, which can be expressed as a frequency change by a factor of $1.059 \mathrm{~Hz}$. All auditory stimuli were ordered randomly, avoiding any musically meaningful or melodic combinations of tones. All of the tones were presented binaurally at a sound pressure level of 70-75 dB via noise-isolating headphones. All participants 
reported that the tones were presented at a clearly audible and comfortable volume. Because the timing of the presentation of the visual items in the lexical decision task was based on the participant's response to the previous trial (i.e., the timing between words was equal to $400 \mathrm{~ms}$ plus the participant's response to the previous trial) and the timing of the tones was two per second, the tones were not presented synchronously with the visual items.

Design A within-subjects design was used in which all participants viewed words and pseudowords under conditions of both irrelevant sound (changing-state tones played through headphones) and silence (no sound played through headphones). The irrelevant-sound and silence trials were blocked into 100 trials per block and were counterbalanced ${ }^{1}$ for starting order across participants. Following the recognition memory task, the same participants performed a serial recall task in which the letters on each trial were presented either in a block of silence or in a separate block of changing-state tones (counterbalanced for starting order across participants).

Procedure Participants were tested individually in a quiet room. Ten practice lexical decision trials were presented in silence to familiarise participants with the task prior to performing any experimental trials. Participants then performed the 200 lexical decision trials (one block of 100 trials with silence, and one block of 100 trials with changing-state auditory tones). Within each block, 50 words and 50 pseudowords were selected randomly and presented visually. Prior to starting the lexical decision task, participants were told that they were participating in a study examining the impact of distracting material on task performance and that their memory for some of the words would be tested on a subsequent recognition memory checklist. They were also told to categorise the visually presented items as words or pseudowords as quickly and accurately as possible (for a word, "press the Z key"; for a pseudoword, "press the M key") while ignoring any auditory material they heard via the headphones. Each visual item remained on screen until a response was made by the participant. Following the participant's response and a 400-ms blank intertrial interval, the next item was displayed.

Immediately after completing both blocks of lexical decision trials, an instruction screen was displayed indicating to participants that a checklist of randomly selected words was about to be shown, some of which had been displayed during the lexical decision task, and that they were to click on any words they remembered seeing during the lexical decision portion of the experiment. The checklist, composed

\footnotetext{
${ }^{1}$ For each experiment, the data were also analysed with an ANOVA that included block order as a between-subjects factor. Block order never interacted with sound condition for any of the memory performance measures in any of the experiments.
}

of 75 randomly selected and simultaneously displayed words ( 25 from the silent block, 25 from the irrelevantsound block, and 25 new-at-test foils), was presented in five columns of 15 words each. Words presented on the checklist were ordered randomly. When a participant clicked on a given word, it was highlighted with a yellow overlay (clicking the word a second time deselected the word and removed this highlight). No minimum or maximum number of responses was imposed, and no time limit was enforced. Clicking on a "next" button ended the checklist session.

Following the checklist session, participants completed the serial recall task. Participants completed 30 trials divided into two counterbalanced blocks of 15 trials each (one in silence, one with irrelevant sound). All participants first completed three trials in silence to familiarise them with the task. Each of the trials consisted of nine consonants ordered randomly and displayed centrally one at a time at a rate of one per second. Irrelevant sound stopped after the presentation of the last consonant in each trial. A 10-s interval followed presentation of the consonant list. Following this interval, an onscreen message instructed participants to write down the letters in the same order in which they had been presented. No time limit was imposed, and participants triggered the start of the next trial with a buttonpress. Participants were told that they would see letters appear on the screen one at a time at a rate of one per second, and that they would need to write them down on the provided paper in the same order in which they were presented. Any sounds heard were to be ignored. At the start of each block, participants were informed whether each block would be an irrelevantsound or a silence block. Sound was presented only during the presentation of consonants.

\section{Results}

Recognition memory performance The mean probabilities of reporting words were .62 in the silent condition and .56 in the tones condition, with an overall false alarm rate of 19 . For each participant, memory sensitivity scores $\left(d^{\prime}\right)$ were calculated separately for words presented in silence and words presented with irrelevant tones (pseudowords were not presented on the test list). ${ }^{2}$ A paired-samples $t$ test performed on the mean $d^{\prime}$ scores as a function of sound condition revealed a significant effect of sound condition, $t$ $(31)=2.56, p=.016, \eta_{\mathrm{p}}^{2}=.174$, in which words presented

\footnotetext{
${ }^{2}$ Because all false alarm items were necessarily new words that had not been presented previously under conditions of silence or noise, false alarms could not be separated into silence and noise conditions. Therefore, the same false alarm value was used for both the silence and noise conditions. Because of this, the sensitivity measure does not change the relative accuracy in the silence and noise conditions for a given participant-it simply reflects their overall ability more accurately.
} 
with irrelevant tones during the lexical decision task were significantly less likely to be reported $\left(d^{\prime}=1.11, S D=0.55\right)$ than words presented in silence $\left(d^{\prime}=1.28, S D=0.52\right)$.

Serial recall performance For each participant, serial recall scores were calculated separately for letters presented in silence and letters presented with tones. For each trial, participants were given a score out of nine (nine letters were presented per trial). A "point" was awarded for each letter correctly recalled in the correct position. Recall proportions were averaged across trials within each sound condition. Across all participants and conditions, the grand mean proportion of correct recalls was $.49(S D=.15)$. Mean recall scores were submitted to a 2 (sound condition) $\times 9$ (serial position) repeated measures analysis of variance (ANOVA). A main effect of serial position was observed, whereby items presented at the beginning or the end of the nine-item set were more likely to be recalled, $F(8,216)=26.33, p<.001, \eta_{\mathrm{p}}{ }^{2}=.494$ (see Fig. 1). The main effect of sound, $F(1,27)=5.88$, $p=.022, \eta_{\mathrm{p}}^{2}=.179$, was also significant, whereby a smaller proportion of items was reported under conditions of irrelevant sound $(M=.47, S D=.15)$ than under conditions of silence $(M=.51, S D=.18)$. The interaction between serial position and irrelevant sound was not significant, $F(8,216)=$ $1.32, p=.235, \eta_{\mathrm{p}}^{2}=.05$.

\section{Discussion}

The results of Experiment 1 showed a significant ISE in a nonserial recognition memory task that did not require the maintenance of serial order information. These same

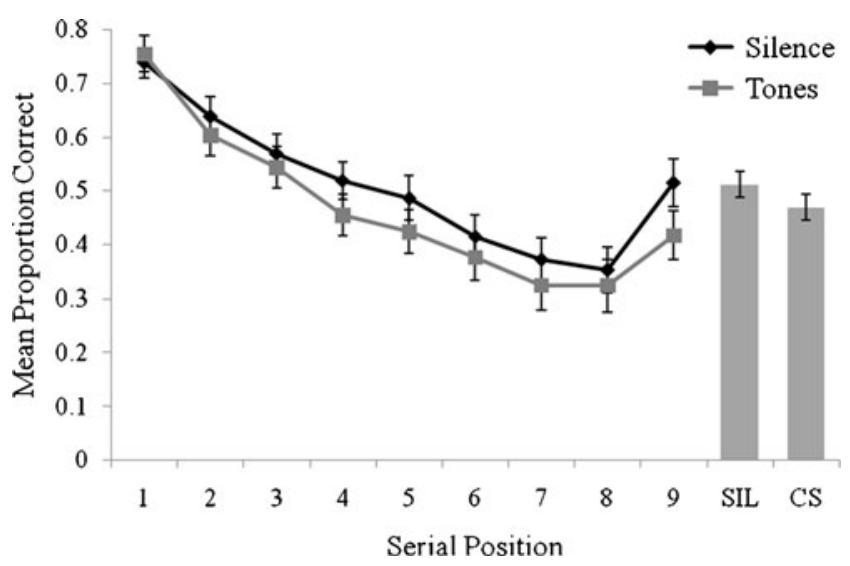

Fig. 1 Memory performance in the serial recall task of Experiment 1. The lines represent the mean proportions of words recalled as a function of irrelevant-sound condition and serial position. The bars represent the mean proportions of words recalled as a function of irrelevant-sound condition: silence (SIL) or changing-state irrelevant sound (CS), collapsed across serial positions. Error bars on the bar graph means represent the standard error of the difference between the two means participants also showed an ISE in a more typical serial recall task using the same irrelevant sound material.

The nonserial recognition results observed here replicated those of LeCompte (1994), in which an ISE was observed in a recognition task in which participants were permitted to report items in any order. Indeed, the difference of $6 \%$ between the irrelevant-sound and silence conditions in the recognition memory task here approximates the difference observed by LeCompte (1994, Exp. 5 C) when using a two-alternative forced choice recognition procedure.

Participants in LeCompte's (1994) experiments viewed word lists that each had 16 or fewer items, and memory was tested in that study after each word list - conditions that are likely to promote serial rehearsal. In contrast, the procedures used in the present experiment did not promote serial rehearsal. Here, a very large word list (100 words) was presented amongst 100 pseudowords, and memory was tested only once, after the presentation of all items. It is very unlikely that participants would attempt serial rehearsal on 100 words while performing a cognitively demanding lexical decision task. However, the possibility of serial rehearsal was addressed further in Experiment 2.

\section{Experiment 2}

Experiment 2 was designed to examine whether the engagement of rehearsal processes could underlie the ISE observed in Experiment 1, in which participants knew that they would need to remember items and may have chosen to engage in serial rehearsal, even though the task and procedures did not promote it. In Experiment 2, we examined the ISE under conditions in which participants were surprised by the nonserial recognition memory test, and were therefore very unlikely to engage in any form of rehearsal. If serial rehearsal processes do underlie the ISE, then when participants are not rehearsing, to-be-remembered visual items presented with concurrent irrelevant, changing-state auditory items should be just as likely to be reported as to-be-remembered visual items presented under a silent control condition. However, if disruption of serial rehearsal and order information is not necessary for the ISE, an ISE might still be observed in the surprise recognition memory test.

\section{Method}

Participants Thirty-four undergraduate students (21 female, 13 male) at Brock University participated in this experiment. Their ages ranged from 18 to 22 years, with a mean of 19.15 years. None of the participants had performed Experiment 1, and none reported knowing about the surprise memory test prior to its presentation during the experiment. 
Design and stimuli The design of the experiment, and the experimental stimuli, were identical to those of Experiment 1 , with the exception that no serial recall task was included.

Procedure Participants completed the lexical decision task and the recognition memory task as in Experiment 1. The only difference was that the participants were not forewarned of the impending memory test, but only became aware of the test when the recognition checklist was presented to them after the completion of both blocks of the lexical decision task. Before completing the lexical decision trials, participants were told that the purpose of the experiment was to examine categorisation speed and accuracy under various forms of distraction, and they were told to ignore any auditory stimuli that they heard.

\section{Results}

Recognition memory performance The mean probabilities of reporting words were .63 in the silent condition and .55 in the tones condition, with an overall false alarm rate of .20 . A paired-samples $t$ test performed on the mean sensitivity scores as a function of sound condition revealed a significant effect of sound condition, $t(33)=3.55, p=.001, \eta_{\mathrm{p}}{ }^{2}=.276$, in which words presented with irrelevant tones during the lexical decision task were significantly less likely to be reported $\left(d^{\prime}=1.07, S D=0.55\right)$ than words presented in silence $\left(d^{\prime}=1.36, S D=0.71\right)$.

\section{Discussion}

The results of Experiment 2 showed a significant ISE in a surprise nonserial recognition memory task that did not require the maintenance of serial order information. Finding an ISE in Experiment 2 replicates Experiment 1, in which an ISE was observed in the same nonserial recognition memory task, but when the memory test was not a surprise. It is very unlikely that rehearsal was employed in the present task, given that 200 items (100 words) were presented during the lexical decision task prior to knowledge of the memory test, under conditions in which participants did not suspect the subsequent memory test, but believed we were studying the effects of irrelevant sound on lexical decision performance.

\section{Experiment 3}

The results of Experiment 2 provide evidence that irrelevant sound can impair memory performance even in the absence of serial rehearsal maintenance of order information. The purpose of Experiment 3 was to replicate the results of Experiment 2 using a different form of irrelevant sound-foreign speech. Foreign speech was the first form of irrelevant sound used
(Colle \& Welsh, 1976) and has been suggested as being a potent irrelevant sound, perhaps due to the fact that speech contains significantly more acoustic changes than any other form of irrelevant sound (Jones \& Macken, 1993, 1995).

\section{Method}

Participants Twenty-eight undergraduate students (21 female, 7 male) at Brock University participated in this experiment. Their ages ranged from 18 to 24 years, with a mean of 19.71 years. None of the participants spoke or understood German, none had participated in Experiment 1 or 2, and no participant reported foreknowledge of the recognition memory test.

Materials All visual stimuli were identical to those of Experiment 1 . However, the irrelevant auditory stimuli were composed of passages from Franz Kafka's A Hunger Artist (Colle \& Welsh, 1976), presented binaurally at a sound pressure level of 70-75 $\mathrm{dB}$ via noise-isolating headphones.

Design The within-subjects design was identical to that used in Experiment 1, but it included irrelevant speech instead of irrelevant tones. As in Experiment 1, the same participants also performed a prototypical serial recall task in which the letters on each trial were presented in a block of silence and in a separate block of irrelevant German speech (counterbalanced for starting order across participants). All participants completed the serial recall task after the recognition task.

Procedure The procedure for the surprise recognition paradigm was identical to that of Experiment 2, and the procedure for the serial recall task was identical to that of Experiment 1.

\section{Results}

Recognition memory performance The mean probabilities of reporting words were .57 in the silent condition and .47 in the speech condition, with an overall false alarm rate of .17. A paired-samples $t$ test performed on the mean sensitivity scores as a function of sound condition revealed a significant effect of sound condition, $t(27)=3.48, p=.002$, $\eta_{\mathrm{p}}{ }^{2}=.310$, in which words presented with irrelevant foreign speech during the lexical decision task were significantly less likely to be reported $\left(d^{\prime}=0.99, S D=0.63\right)$ than words presented in silence $\left(d^{\prime}=1.27, S D=0.57\right)$.

Serial recall performance Serial recall scores were calculated as in Experiment 1. Across all participants and conditions, the grand mean proportion of correct recall was .46 $(S D=.11)$. Mean recall scores were submitted to a 2 (sound condition) $\times 9$ (serial position) repeated measures ANOVA. A main effect of 
serial position was observed, whereby items presented at the beginning or end of the nine-item set were more likely to be recalled, $F(8,216)=44.59, p<.001, \eta_{\mathrm{p}}{ }^{2}=.623$ (see Fig. 2). The main effect of sound, $F(1,27)=64.51, p<.001$, $\eta_{\mathrm{p}}^{2}=.705$, was also significant, whereby items that were presented under conditions of irrelevant foreign speech were less likely to be reported than items presented in silence. The interaction between serial position and irrelevant speech was not significant, $F(8,216)=.68, p=.71, \eta_{\mathrm{p}}{ }^{2}=.025$.

\section{Discussion}

The results of Experiment 3 replicated those observed in Experiments 1 and 2. As in Experiment 2, a significant ISE was observed in a surprise nonserial recognition memory task that did not encourage the use of a rehearsal strategy or the preservation of serial order information.

As in Experiment 1, the results from the prototypical serial recall task showed the expected ISE. Indeed, the ISE observed in the present experiment $\left(\eta_{\mathrm{p}}^{2}=.705\right)$ was statistically larger than the ISE observed in Experiment 1 $\left(\eta_{\mathrm{p}}{ }^{2}=.179\right)$, which used the same serial recall task, but with changing-state tones instead of irrelevant speech. This pattern is consistent with previous research showing that irrelevant speech is at least as disruptive to serial recall performance as changing-state tones, if not more so (e.g., Jones \& Macken, 1993, 1995).

\section{Experiment 4}

It is very likely that serial rehearsal did not underlie the recognition memory results observed in Experiments 2 and

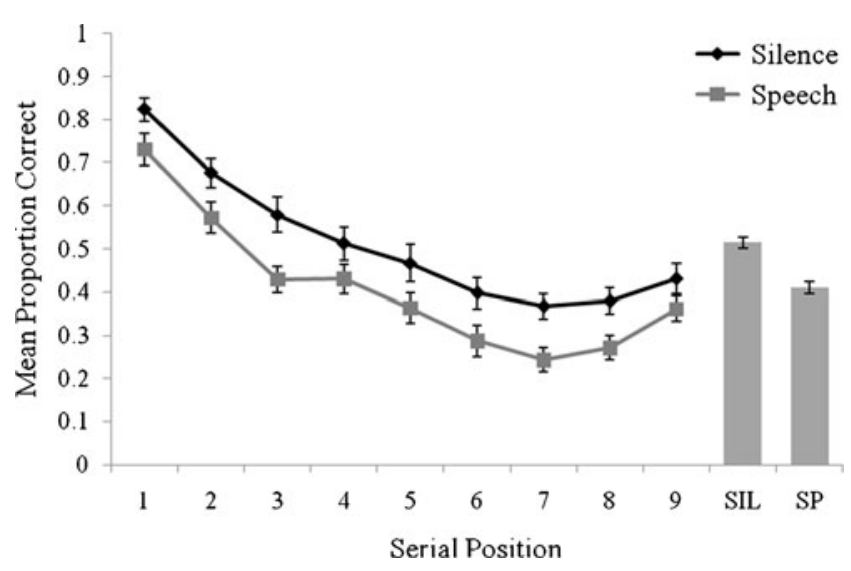

Fig. 2 Memory performance in the serial recall task of Experiment 3. The lines represent the mean proportions of words recalled as a function of irrelevant-sound condition and serial position. The bars represent the mean proportions of words recalled as a function of irrelevant-sound condition: silence (SIL) or irrelevant foreign speech (SP), collapsed across serial positions. Error bars on the bar graph means represent the standard error of the difference between the two means
3 , and as such, it is tempting to assume that those results derived from a disruption of item, not order, information. However, a direct test of this assumption was still needed. This was accomplished in Experiment 4 by comparing surprise recognition memory performance for changing-state and steady-state irrelevant sounds.

Jones and Macken (1993, 1995; see also Jones, Madden, \& Miles, 1992) demonstrated that changing-state irrelevant stimuli (e.g., a sequence of tones composed of different pitches) are significantly more disruptive to serial recall than are steady-state irrelevant stimuli (e.g., a single tone repeated at regular temporal intervals). ${ }^{3}$ According to Jones and Macken (1993, 1995), irrelevant changing-state stimuli are markedly more disruptive to a primary task than are irrelevant steadystate stimuli because of the increased confluence of irrelevant order cues with serial rehearsal processes. The authors argued that confusion in seriation processes common to both irrelevant stimuli and the to-be-remembered items underlies this changing-state effect.

In Experiment 4, we examined whether the increased disruptive nature of changing-state stimuli (over steady-state stimuli) could be observed in our nonserial surprise recognition task. If disruption of identity, but not order, information underlay the ISE results observed in the surprise recognition memory test in Experiments 2 and 3, then the visual items presented now with concurrent irrelevant steady-state tones should elicit impaired recognition memory equal to that observed with visual items presented with concurrent irrelevant changingstate tones. However, if disruption of order, but not identity, information underlay the ISE results observed in with the surprise recognition memory test in Experiments 2 and 3, then the visual items presented now with concurrent irrelevant steady-state tones should elicit no impairment in recognition memory, whereas visual items presented with concurrent irrelevant changing-state tones should elicit the same recognition memory impairment observed in Experiment 2.

Method

Participants Forty-four Brock University undergraduate students (33 female, 11 male) participated in this experiment. Their ages ranged from 18 to 28 years, with a mean of 20.20 years. None of the participants had participated in any of the previous experiments.

Materials All visual stimuli were identical to those of Experiment 2. The irrelevant auditory stimuli were tones, as in Experiment 2, but they varied in presentation across conditions.

\footnotetext{
${ }^{3}$ Even when presented at a constant rate, sound sequences are classified as "changing-state" as long as each successive item is different from the preceding item in at least one acoustic aspect (e.g., pitch or timbre).
} 
The changing-state stimuli and their presentation conditions were the same as the tone presentations in Experiment 2. The steady-state distractors consisted of the repeated presentation of a single auditory tone, selected randomly on each trial from the same set of tones used for the changing-state stimuli. This single repeated tone was presented for the same duration, at the same rate, and under the same conditions as the changing-state tones (i.e., the only difference between the changingstate condition and the steady-state condition was whether the pitch of the tone changed from tone to tone, or stayed constant for all tones).

Design and procedure The within-subjects design was identical to that used in Experiment 2, but it included an additional steady-state sound condition. All participants performed 216 lexical decision trials (108 words, 108 pseudowords) in which the items were divided equally into three 72-trial blocks: silence, steady-state tones, and changingstate tones. Each block contained 36 words and 36 pseudowords, randomly selected and displayed. Each participant completed all three blocks in an order determined in advance by permutation $(\mathrm{ABC}, \mathrm{BCA}, \mathrm{CAB}, \mathrm{CBA}, \mathrm{ACB}$, $\mathrm{BAC}$ ), such that the irrelevant-sound condition order was counterbalanced every six participants. The surprise recognition checklist was composed of 27 words from each condition block, as well as 27 words that were new-at-test (foils), for a total of 108 words.

\section{Results}

Surprise nonserial recognition performance The mean probabilities of reporting words were .59 for silence, .53 for changing-state tones, and .53 for steady-state tones, with an overall false alarm rate of .20. For each participant, memory sensitivity scores $\left(d^{\prime}\right)$ were calculated separately for words presented in silence, with irrelevant steady-state tones, and with irrelevant changingstate tones. Across all participants, the grand mean sensitivity score was $.35(S D=.16)$.

The mean sensitivity scores were submitted to a one-way (sound condition: silence vs. steady-state vs. changing-state irrelevant sound) repeated measures ANOVA. A main effect of sound condition was observed, $F(2,86)=4.12, p=.020$, $\eta_{\mathrm{p}}{ }^{2}=.087$ (see Fig. 3). Follow-up planned paired-samples $t$ tests indicated that, relative to the silence condition, sensitivity scores were lower in both the changing-state irrelevantsound, $t(43)=2.33, p=.024, \eta_{\mathrm{p}}{ }^{2}=.113$, and the steady-state irrelevant-sound, $t(43)=2.84, p=.007, \eta_{\mathrm{p}}^{2}=.158$, conditions, but that the two irrelevant-sound conditions did not differ from each other, $t(43)=0.94, p=.926, \eta_{\mathrm{p}}{ }^{2}=.000$. The same pattern was also observed using Tukey's HSD test, which includes appropriate alpha correction (for the silence

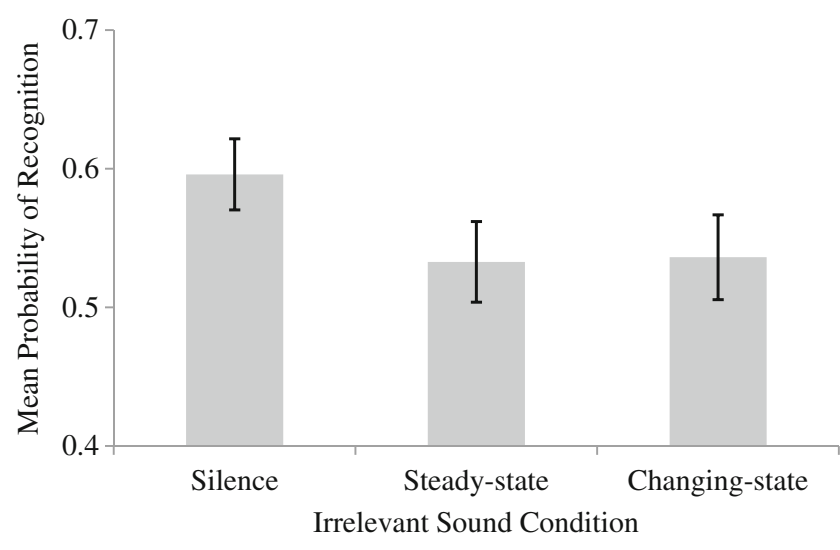

Fig. 3 Mean probabilities of correct recognition on the surprise recognition memory test in Experiment 4 as a function of irrelevant-sound condition. Error bars represent the standard error of the mean for each condition

vs. changing-state and silence vs. steady-state irrelevantsound comparisons, both $p \mathrm{~s}<.05$; for changing-state vs. steady-state, $p>.05$.)

\section{Discussion}

The results of Experiment 4 replicated the results of Experiment 2, in that a significant effect of changing-state irrelevant sound on memory performance was again observed in a surprise recognition memory task that discouraged the use of a rehearsal strategy and did not require the maintenance of serial order information. However, the finding of an equally large ISE for the changing-state and steady-state conditions provided evidence that any form of irrelevant sound, whether changing-state or steady-state, can disrupt memory performance in this surprise nonserial recognition task. The changing-state effect (i.e., greater ISE for irrelevant auditory changing-state stimuli than for irrelevant auditory steady-state stimuli) observed in serial recall tasks is thought to result from the ability of changing-state stimuli to obligatorily impose their own order, thereby confusing the order information for primary task stimuli (e.g., Jones \& Macken, 1993). In contrast, steady-state stimuli generate little order information and are therefore minimally disruptive to order information in the primary task (e.g., Jones \& Macken, 1993). If this logic is correct, the present results showing equivalent impairment for changing-state and steady-state auditory stimuli provide evidence that order information is not impaired in the surprise recognition memory task used here, but rather identity/perceptual information.

It is tempting to suggest that a stronger manipulation of steady-state versus changing-state irrelevant sound would have generated a pattern of results in which the ISE was larger with changing-state sounds. One could argue that the use of a single repeated tone still possesses changing-state properties. 
For example, the onset and offset of each tone, although regular, could be interpreted as a changing property. A more steady stimulus could be white or pink noise, which is a more continuous sound source and does not contain any abrupt "change." However, earlier research contrasting serial digit recall performance in silence versus white noise bursts (which also have an "on then off" pattern) yielded null results (Jones \& Macken, 1993; Salamé \& Baddeley, 1982). Nonetheless, it may be beneficial for future studies to examine irrelevant white/pink noise as the steady-state irrelevant-sound source.

\section{Interexperiment analyses}

\section{Recognition memory performance}

Across all four experiments, a compelling effect of irrelevant sound on recognition memory performance emerged. To examine whether the magnitude of the ISE effect changed across experiments, a 2 (within: irrelevant changing-state sound vs. silence) $\times 4$ (Experiment 1-4) mixed-model ANOVA was employed to examine the recognition memory scores. ${ }^{4}$ The results showed a main effect for irrelevant sound across all experiments, $F(1,134)=33.92, p<.001, \eta_{\mathrm{p}}{ }^{2}=.202$. Neither the main effect of experiment, $F(3,134)=0.40$, $p=.753, \eta_{\mathrm{p}}{ }^{2}=.009$, nor the Sound Condition $\times$ Experiment interaction, $F(3,134)=0.60, p=.619$, $\eta_{\mathrm{p}}^{2}=.013$, was significant. See Fig. 4, showing the ISE in each experiment, including in the steady-state condition of Experiment 4 (not included in this analysis). Taken together, these results show that ISE magnitude using our surprise recognition memory task was not modulated by whether or not participants were aware of the impending memory test, whether the irrelevant sound was composed of foreign speech or tones, or whether the nature of the irrelevant sound was changing-state or steady-state.

\section{Lexical decision times}

In all four experiments, participants were presented with tobe-remembered items via an online lexical decision task. In addition to providing a cover story for the incidental presentation of test items, it provided the benefit of calculating

\footnotetext{
${ }^{4}$ To maintain a consistent comparison across experiments, and because in Experiment 4 performance in the steady-state sound condition was equivalent to that in the changing-state sound condition, in all cases only the comparison between the changing-state sound condition and silence was included in the analysis. The steady-state sound condition was excluded from this analysis because it was a within-subjects manipulation in Experiment 4; nonetheless, it is included in Fig. 4 for a visual comparison across all conditions and experiments.
}

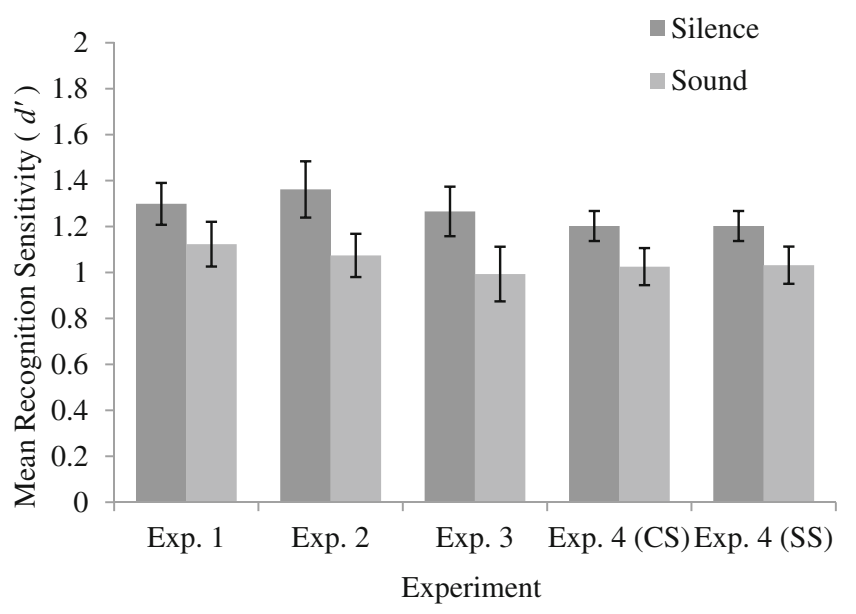

Fig. 4 Mean recognition sensitivity scores, represented by $d^{\prime}$, for the silence and irrelevant-sound conditions. Two contrasts from Experiment 4 are included in this figure (but not in the interexperiment statistical analysis) to compare both changing- and steady-state irrelevant sounds with silence. Error bars represent the standard error of each mean within each experiment

online response time (RT) measures for items under conditions of irrelevant sound versus silence.

All lexical decision RTs were based on trials in which the item was classified correctly. Within each experiment, correct RTs were subjected to an outlier elimination procedure performed separately for each combination of participant, letter string (word/pseudoword), and sound condition. RTs greater than three standard deviations from the mean were removed, and these accounted for fewer than $2 \%$ of all trials in each of the experiments.

To test for the possibility that irrelevant sound impacted online performance, a 2 (within: changing-state sound vs. silence) $\times 2$ (within: word vs. pseudoword) $\times 4$ (between: experiment) mixed-model ANOVA was employed to examine the RTs to visual lexical decision items. ${ }^{5}$ The results revealed a main effect of irrelevant sound, $F(1,134)=14.79, p<.001$, $\eta_{\mathrm{p}}^{2}=.099$, such that faster RTs were observed under conditions of irrelevant sound $(M=659, S D=88)$ than under silence $(M=693, S D=128)$. A main effect was also observed for item type, $F(1,134)=110.14, p<.001, \eta_{\mathrm{p}}{ }^{2}=.451$, such that faster RTs were observed for words $(M=629, S D=88)$ than for pseudowords $(M=722, S D=128)$. The betweensubjects effect of experiment was nonsignificant, $F(1,3)=$ $.696, p=.556, \eta_{\mathrm{p}}^{2}=.015$, as were all of the interactions, all $p \mathrm{~s}>.12$, including the Experiment $\times$ Sound Condition interaction, $F<1$. The lexical decision results are discussed further in the General Discussion.

\footnotetext{
${ }^{5}$ In keeping with the comparison parameters discussed in terms of recognition sensitivity between the experiments, the steady-state irrelevant-sound condition of Experiment 4 was also excluded from these analyses.
} 


\section{General discussion}

To summarise, Experiment 1 revealed that the ISE (impaired memory performance associated with the presence of irrelevant background sound relative to silence) can be observed when participants are forewarned of a nonserial recognition task in which maintenance of order information is not critical to the task. The same participants also showed an ISE in a more prototypical serial recall task. In Experiment 2, we tested for the ISE in the same nonserial recognition task as in Experiment 1 , but the participants were not made aware of the impending memory test until after completing the lexical decision task. An ISE was again observed with this surprise nonserial recognition task. Because 200 lexical items were presented in the lexical decision task, and participants were surprised by the memory test, they were very unlikely to have rehearsed or employed strategies for the maintenance of serial order representations. In Experiment 3, we tested for the ISE under conditions similar to those of Experiment 2, except that instead of irrelevant auditory tones, the irrelevant sound was composed of foreign speech. The results replicated those of Experiment 2 and indicated that irrelevant foreign speech leads to an ISE in surprise nonserial recognition memory. The same participants also showed an ISE in a more prototypical serial recall task using the same foreign speech. For Experiment 4, we examined whether disruption of order and/or identity representations underlay the recognition memory results observed with irrelevant sound in Experiments 1-3 by testing for an ISE under conditions of changing-state and steady-state irrelevant tones. The results indicated that surprise nonserial recognition memory performance was impaired equally under both irrelevantsound conditions as compared to a silent control, suggesting an impairment of identity, not of order, representations.

The results of the present experiments contrast with those of Salamé and Baddeley (1990), who observed no ISE in a free recall task. The results of Experiment 1 do replicate LeCompte's (1994) findings of an ISE using several nonserial recognition paradigms. Experiments 2-4 of the present study extended LeCompte's (1994) findings by demonstrating an ISE in a nonserial recognition paradigm in which participants were surprised by the memory test. The addition of surprise was an important element of the study, given that it dramatically reduced the likelihood that participants would rehearse the lexical decision items. It was critical to rule out the possibility of rehearsal, because participants who know a memory test is coming may use a serial rehearsal strategy even in a task in which order information is not required (Beaman \& Jones, 1997). Indeed, as noted in the introduction, Beaman and Jones provided evidence that participants in LeCompte's (1994) study may have been using a serial rehearsal strategy. LeCompte's use of 12- to 16-item lists with a recognition test after each list may also have increased the likelihood that his participants would use a serial rehearsal strategy. The present study rendered rehearsal and maintenance of order information extremely unlikely through the use of a cognitively demanding cover task (lexical decision) and a long (200-item) list of potential memory items, as well as by incorporating the element of surprise.

\section{Disruption of order versus identity codes}

Typically, the ISE is examined with serial recall tasks in which the same set of items are used on every trial. Participants are either told or rapidly come to learn the identities of the items on each trial. Thus, the only errors that are observed are order errors. In this way, typical serial recall tasks isolate the impact of irrelevant sound to its effect on order information, not identity information.

In addition to the handful of free recall and recognition tasks discussed above, the issue of whether irrelevant sound could impact item identity, independent of order information, has been examined previously using the missing-item task (Beaman \& Jones, 1997; Jones \& Macken, 1993; LeCompte, 1996). In this task, participants are presented with all but one of a well-known set of items (e.g., days of the week or the digits 0-9), after which they are required to report the missing item. Performance in this task is sometimes unimpaired by irrelevant sound (e.g., Beaman \& Jones, 1997; Jones \& Macken, 1993) or sometimes shows a small, but reliable, ISE (Beaman \& Jones, 1997, Exp. 1; LeCompte, 1996). Jones and Macken (1993) described the missing-item task as relying heavily on the retention of item but not order information. They have interpreted the null or inconsistent ISEs with the missing-item task as evidence that irrelevant sound does not impair identity information.

In contrast to the conclusion of Jones and Macken (1993), the present results show that irrelevant sound can reliably impair memory for item identity, independent of order information, when a surprise recognition memory test is used. Irrelevant-sound effects were reliably observed in each of the four nonserial recognition memory experiments reported here. Furthermore, finding an ISE with both steady-state and changing-state sounds in Experiment 4 suggested that identity, not order, information underlay the ISEs observed here. Thus, the present evidence suggests that it is possible for irrelevant sound to impair identity information alone. When the results of the present study are combined with those of previous studies using a serial recall task with a small, finite set of items (e.g., Beaman \& Jones, 1997; LeCompte, 1994), there is evidence that the ISE can reflect impairment of identity information and/or order information.

Implications for models of the ISE

Many models have been offered to account for the effect of irrelevant sound on memory performance. Common to several 
of these models is the notion that disruption of order information underlies the ISE. For example, according to the primacy model (Page \& Norris, 1998), order information is stored and referenced via the activation strengths of successive list items. Activation strength is said to decrease linearly for each item in a list, to form a so-called primacy gradient. Serial output is generated by the repeated referencing of to-be-recalled items in ranked order of activation strength. Irrelevant sound is thought to add noise to the primacy gradient, thus blurring the distinction between order cues. Similarly, the O-OER model (Jones, 1993) stresses that the memory impairment reflected in the ISE is based on the disruption of order information and serial rehearsal processes. However, these accounts, and other models that rely solely on impairment of order information to explain the ISE, are unable to explain the present results, given that the series of experiments here did not require participants to serially report to-be-remembered items, and indeed participants did not even know that they would need to remember the items. Furthermore, the finding that steady-state and changing-state irrelevant sound impaired surprise recognition memory performance equally provides evidence that order information was not impaired in the present studies.

The feature model (Neath, 1999) posits that impairment of phonological codes underlies the ISE, so that the degree to which irrelevant and relevant stimuli match acoustically is predictive of the level of disruption. However, this model cannot readily account for the present results, among others, given that our experiments, along with several previous ones (e.g., Beaman \& Jones, 1997; Jones \& Macken, 1993; Macken, Tremblay, Houghton, Nicholls, \& Jones, 2003), have shown an ISE when the irrelevant sound was composed of nonspeech auditory tones that shared few acoustic features with the verbal to-be-remembered items.

Broadbent's (1984) model, in which the ISE was said to result from a breakdown during encoding into working memory, could account for the present finding of an ISE for identity information, as could Cowan's $(1995,1999)$ embeddedprocesses model of working memory, in which the ISE was said to result from the redirection of the focus of attention away from the primary stimuli or task. In both of these models, stimuli must first be detected, then encoded, before they can be stored in memory. These models do not rely on a disruption of order information or of rehearsal processes to explain the ISE. Instead, they suggest that items held in the focus of attention at the moment of distraction are only weakly represented in short-term (and long-term) memory. Thus, to the extent that the primary task involves the preservation of item information for later recall (whether this preservation is purposeful or incidental), interference by irrelevant stimuli should reduce subsequent memory performance.

However, these broader attentional models have difficulty accounting for the full range of previous empirical ISE results.
For example, if the ISE is driven only by disruptions of attentional processes, one might reasonably expect the effect to habituate over time. Indeed, Banbury and Berry (1997) provided evidence that, despite an initial effect of irrelevant sound, memory for prose improves after prolonged exposure to irrelevant speech within an experimental session. Despite a clear ISE during the first half of their experimental session, the effect of irrelevant sound decreased by the second half if participants were continuously exposed to the same irrelevant sound during the intertrial interval before the presentation phase of the second half. However, this has not been a typical case (e.g., Hughes et al., 2005; Jones, Macken, \& Mosdell, 1997; Tremblay \& Jones, 1998); extended exposure to irrelevant sound does not typically diminish the ISE within an experimental session or between sessions in which the same irrelevant sounds are presented (e.g., Ellermeier \& Zimmer, 1997; Hellbrück, Kuwano, \& Namba, 1996). It is important to note that, in the case of Banbury and Berry's experiment, if no sound was played during the interval between trials, the ISE remained. This pattern of dishabituation may be reflected in more typical ISE studies in which serial recall trials are interleaved with silent intervals (e.g., before or during the recall phase and the presentation phase of the next trial). In future studies, researchers may want to examine memory performance under conditions of continuous presentation of irrelevant sound throughout the entire experimental session. Nonetheless, a purely attentional-encoding account of the ISE also has difficulty explaining the finding that irrelevant sound presented only during a retention phase following the presentation of to-be-remembered material still impairs memory performance (Macken, Mosdell, \& Jones, 1999).

It is clear that irrelevant sound can impair the correct report of items when the sound is presented only during a retention interval (Macken et al., 1999) or is presented at encoding and no rehearsal is performed (the present results). Therefore, disruption of serial rehearsal may be sufficient to observe an ISE, but the present results demonstrate that it is not necessary. It is clear that a complete model of the ISE needs to account for the disruptive impact of irrelevant sound on identity codes at encoding and/or during retention of the representations, even under conditions in which active rehearsal and maintenance of order information may be absent, as well as to account for results showing that presentation of irrelevant sounds during rehearsal is sufficient to impair order information, resulting in an ISE (Macken et al., 1999).

One could instead suggest that a changing-state effect is the true hallmark of the ISE (e.g., Jones \& Macken, 1993), and as such, the present results do not show the ISE per se, but rather a separate irrelevant-sound phenomenon. If that were the case, models such as the embedded-processes model of Cowan $(1995,1999)$ would not need to account for results observed with an ISE based on order impairment and could account nicely for the present results showing 
identity impairments with irrelevant sound. However, on the grounds of parsimony, we suggest that invoking separate effects of irrelevant sound for each task type is superfluous. Instead, a more general effect of irrelevant sound may have the potential to disrupt both item and order information. In tasks that require the retention of item but not order information (such as those used here), the ISE can be observed in deficits of item information. In contrast, in tasks requiring serial recall of a small learned set (e.g., Jones \& Macken, 1993), rehearsal can be impaired, and the ISE can be observed in deficits of order information.

Recall that the perceptual-gestural model stresses that the memory impairment reflected in the ISE is based on two related components, one perceptual and one gestural (Jones et al., 2006; Jones et al., 2004). The gestural component reflects the nature of the reporting strategy. In tasks that require serial reporting, participants may engage a gestural strategy (e.g., subvocal articulation or counting on one's fingers) that mirrors the reporting conditions. However, in all of the experiments reported here, participants were not required to serially report to-be-remembered items. Thus, disruptions within the gestural component of this model cannot sufficiently explain the present data. However, the ISE reported here could be based on disruptions of the perceptual organisation of incoming stimuli (i.e., the perceptual component). According to the perceptual-gestural model, all incoming stimuli are automatically organised in a way that directly reflects the nature of the external stimuli. For example, if the to-be-remembered stimuli are presented alphabetically, the internal representations of those items will also be organised alphabetically. Likewise, if the inherent organisation of the to-be-remembered stimuli is unclear, reference to its organisation will be accordingly unclearunless the perceiver makes a deliberate effort (embodied within the gestural component) to reorganise the incoming stimuli into a meaningful order. The impairment of identity information observed here may result, not from the perturbation of output planning, but via the means through which the items are organised. These nonphonological representations are subject to intrusions from auditory speech or nonspeech stimuli, given that obligatory access is granted to both auditory speech and nonspeech stimuli (cf. Bregman, 1990). Irrelevant sound may perturb the perceptual organisation of all incoming stimuli, which, as described by Jones et al. (2004), will increase the amount of disruption. This would also be consistent with the results from Experiment 4, which suggest that the ISE observed in the present series of experiments is not triggered by changing-state sound, but rather by irrelevant sound more generally.

Hughes et al. (2005) proposed the duplex-mechanism model, which includes two separate mechanisms by which irrelevant sound can interfere with memory performance. The first mechanism deals with the competition between relevant and irrelevant seriation processes, in which order information from the irrelevant sound impairs order information for the to-be-remembered material. The second mechanism centres on attentional orienting to irrelevant auditory distractors, which interrupts the encoding of target items, such that to-be-remembered target items presented while attention is oriented away from the primary task are poorly encoded and reported. Hughes et al. used this model to dissociate the typical ISE from the so-called deviation effect, in which infrequent auditory stimuli that violate an established irrelevant acoustic pattern capture attention at the cost of performance in a primary memory task. Specifically, the ISE is attributed to the first mechanism, whereas memory costs incurred under the umbrella of the deviation effect are typically explained in terms of the second (attentional) mechanism. However, although Hughes et al. did not suggest that the ISE could result from either the first or the second mechanism, if one made this assumption (in which the first seriation mechanism accounted for order impairments and the second accounted for identity impairments), this model could explain the present results as well as accommodating more traditional ISE findings.

The extent to which the deviation effect is related to the ISEs observed in the present series of experiments is indeed unclear and worth testing, although the results of Experiment 4 make it seem more likely that they would be distinct. The full potential of the duplex-mechanism account to explain the present results may depend on future studies that test whether or not the deviation effect is related to the surprise nonserial recognition memory effect observed here.

\section{Lexical decision performance}

Online measurements of lexical decision performance did not show impairments on irrelevant-sound blocks. Indeed, in the present work irrelevant sound tended to speed up participants' lexical decisions for words and pseudowords, not slow them down. One might reasonably expect that irrelevant sound would have slowed participants down due to some form of attentional redirection away from the primary task (e.g., Cowan, 1995; Page \& Norris, 1998). Why, then, might irrelevant sound impair recognition memory performance relative to the silent control condition, but not impair lexical decision RTs? A possible explanation lies in the fact that the lexical decision task could be performed without encoding the words into a more durable memory representation, while such encoding was required for successful memory performance. Whether or not participants were aware of the impending memory test, only items that were successfully represented in memory would be able to support later recognition memory performance. Irrelevant sound may impair processes involved in this representation, whether such maintenance for a later memory test is purposeful (Exp. 1) or incidental (Exps. 2, 3, and 4). In 
contrast, successful performance on the lexical decision trials required only activation of existing nodes in semantic memory (or a failure to activate a node, in the case of pseudowords) and the attentional resources to translate the presence/absence of activation into a response selection of the appropriate key. No durable memory representation of the lexical item was required for successful lexical decision performance. Following this logic, one could suggest that the ISE that has been observed here is constrained to resource-demanding intermediary processes such as memory encoding, as opposed to the degradation of earlier perceptual representations.

While the explanation above can explain why lexical decision RTs were not slowed by irrelevant sound, it cannot explain why they were faster with irrelevant sound. Smith (2010) also found that irrelevant sound or speech decreases response times to decision stimuli. Smith noted the possibility that irrelevant sound may force a perceiver to invest more attentional resources in the primary task, as a byproduct of reducing the resources oriented to the irrelevant material. However, this explanation is speculative and does not appear consistent with the decreased item memory observed here for words presented with irrelevant sound. Other possible alternative explanations could be that the irrelevant sound increases alertness or that participants use the tone to pace their RTs, but these are also speculative.

\section{Conclusion}

The present series of experiments is the first, to our knowledge, to test for an ISE in a surprise nonserial recognition task. The present results show that the ISE can be observed in the absence of the rehearsal and maintenance of order information and with both steady-state and changing-state sound, suggesting that the effect can result from an impairment of identity codes, as opposed to order information. Several other studies of the ISE have shown that irrelevant changing-state sound (but not steady-state sound) impairs order information in serial recall tasks. Thus, the detrimental effects of irrelevant sound appear to have two sources, one that reduces the fidelity of identity codes in memory, and another that reduced the fidelity of order information during serial rehearsal. These two impairments are both sufficient, but neither is necessary, to observe the ISE.

Author note This work was funded by grants from the National Sciences and Engineering Research Council of Canada (NSERC), the Canadian Foundation for Innovation (CFI), and the Ontario Innovation Trust (OIF) to the second author, and by an NSERC Canada Graduate Scholarship to the first author. We thank Matthew Goldhawk for his assistance with data collection.

\section{References}

Banbury, S., \& Berry, D. C. (1997). Habituation and dishabituation to speech and office noise. Journal of Experimental Psychology: Applied, 3, 181-195. doi:10.1037/1076-898X.3.3.181

Beaman, C. P., \& Jones, D. M. (1997). Role of serial order in the irrelevant speech effect: Tests of the changing-state hypothesis. Journal of Experimental Psychology: Learning, Memory, and Cognition, 23, 459-471. doi:10.1037/0278-7393.23.2.459

Bregman, A. S. (1990). Auditory scene analysis: The perceptual organization of sound. Cambridge, MA: MIT Press.

Broadbent, D. (1984). The Maltese cross: A new simplistic model for memory. Behavioral and Brain Sciences, 7, 55-94. doi:10.1017/ S0140525X00026121

Colle, H. A., \& Welsh, A. (1976). Acoustic masking in primary memory. Journal of Verbal Learning and Verbal Behavior, 15, 17-31. doi:10.1016/S0022-5371(76)90003-7

Cowan, N. (1995). Attention and memory: An integrated framework. New York, NY: Oxford University Press.

Cowan, N. (1999). An embedded-processes model of working memory. In A. Miyake \& P. Shah (Eds.), Models of working memory (pp. 62-101). Cambridge, U.K.: Cambridge University Press.

Ellermeier, W., \& Zimmer, K. (1997). Individual differences in susceptibility to the "irrelevant speech effect. Journal of the Acoustical Society of America, 102, 2191-2199. doi:10.1121/1.419596

Hellbrück, J., Kuwano, S., \& Namba, S. (1996). Irrelevant background speech and human performance: Is there long-term habituation? Journal of the Acoustical Society of Japan, 17, 239-247.

Hughes, R. W., Vachon, F., \& Jones, D. M. (2005). Auditory attentional capture during serial recall: Violations at encoding of an algorithmbased neural model? Journal of Experimental Psychology: Learning, Memory, and Cognition, 31, 736-749. doi:10.1037/02787393.31.4.736

Jones, D. M. (1993). Objects, streams and threads of auditory attention. In A. D. Baddeley \& L. Weiskrantz (Eds.), Attention: Selection, awareness and control (pp. 87-104). Oxford, U.K.: Oxford University Press.

Jones, D. M., Hughes, R. W., \& Macken, W. J. (2006). Perceptual organization masquerading as phonological storage: Further support for a perceptual-gestural view of short-term memory. Journal of Memory and Language, 54, 265-281. doi:10.1016/j.jml.2005.10.006

Jones, D. M., \& Macken, W. J. (1993). Irrelevant tones produce an irrelevant speech effect: Implications for phonological coding in working memory. Journal of Experimental Psychology: Learning, Memory, and Cognition, 19, 369-381. doi:10.1037/02787393.19.2.369

Jones, D. M., \& Macken, W. J. (1995). Phonological similarity in the irrelevant speech effect: Within- or between-stream similarity? Journal of Experimental Psychology: Learning, Memory, and Cognition, 21, 103-115. doi:10.1037/0278-7393.21.1.103

Jones, D. M., Macken, W. J., \& Mosdell, N. (1997). The role of habituation in the disruption of recall by irrelevant sound. British Journal of Psychology, 88, 549-564.

Jones, D. M., Macken, W. J., \& Nicholls, A. P. (2004). The phonological store of working emeory: Is it phonological and is it a store? Journal of Experimental Psychology: Learning, Memory, and Cognition, 30, 656-674. doi:10.0137/0278-7393.30.3.656

Kučera, H., \& Francis, W. N. (1967). Computational analysis of present day American English. Providence, RI: Brown University Press.

LeCompte, D. (1994). Extending the irrelevant speech effect beyond serial recall. Journal of Experimental Psychology: Learning, Memory, and Cognition, 20, 1396-1408. doi:10.1037/02787393.20.6.1396

LeCompte, D. C. (1996). Irrelevant speech, serial rehearsal, and temporal distinctiveness: A new approach to the irrelevant speech effect. 
Journal of Experimental Psychology: Learning, Memory, and Cognition, 22, 1154-1165. doi:10.1037/0278-7393.22.5.1154

Macken, W. J., Mosdell, N., \& Jones, D. M. (1999). Explaining the irrelevant-sound effect: Temporal distinctiveness or changing state? Journal of Experimental Psychology: Learning, Memory, and Cognition, 25, 810-814. doi:10.1037/0278-7393.25.3.810

Macken, W. J., Tremblay, S., Houghton, R. J., Nicholls, A. P., \& Jones, D. M. (2003). Does auditory streaming require attention? Evidence from attentional selectivity in short-term memory. Journal of Experimental Psychology: Human Perception and Performance, 29, 43-51. doi:10.1037/0096-1523.29.1.43

Neath, I. (1999). Modelling the disruptive effects of irrelevant speech on order information. International Journal of Psychology, 34, $410-418$.

Page, M. P. A., \& Norris, D. (1998). The primacy model: A new model of immediate serial recall. Psychological Review, 105, 761-781. doi:10.1037/0033-295X.105.4.761-781
Salamé, P., \& Baddeley, A. (1982). Disruption of short-term memory by irrelevant speech: Implications for the structure of working memory. Journal of Verbal Learning and Verbal Behavior, 21, 150-164. doi:10.1016/S0022-5371(82)90521-7

Salamé, P., \& Baddeley, A. D. (1990). The effects of irrelevant speech on immediate free recall. Bulletin of the Psychonomic Society, 28, 540-542.

Schneider, W., Eschman, A., \& Zuccolotto, A. (2002). E-Prime user's guide. Pittsburgh, PA: Psychology Software Tools.

Smith, P. A. (2010). Ageing, auditory distraction, and grammaticality judgement. Aphasiology, 24(11), 1342-1353. doi:10.1080/02687 030903490533

Tremblay, S., \& Jones, D. M. (1998). Role of habituation in the irrelevant sound effect: Evidence from the effects of token set size and rate of transition. Journal of Experimental Psychology: Learning, Memory, and Cognition, 24, 659-671. doi:10.1037/ 0278-7393.24.3.659 